\title{
Hereditary diffuse gastric cancer in two families: A case report
}

\author{
IRENE FEROCE ${ }^{1}$, DAVIDE SERRANO $^{1}$, ROBERTO BIFFI $^{2}$, BRUNO ANDREONI $^{3}$, \\ VIVIANA GALIMBERTI ${ }^{4}$, ANGELICA SONZOGNI ${ }^{5}$, LUCA BOTTIGLIERI $^{5}$, EDOARDO BOTTERI ${ }^{6}$, \\ CRISTINA TROVATO $^{7}$, MONICA MARABELLI $^{8}$, GUGLIELMINA NADIA RANZANI $^{8}$ and BERNARDO BONANNI ${ }^{1}$
}

Divisions of ${ }^{1}$ Cancer Prevention and Genetics, ${ }^{2}$ Abdomino-Pelvic Surgery and ${ }^{3}$ Palliative Care; ${ }^{4}$ Unit of Molecular Senology; Divisions of ${ }^{5}$ Pathology, ${ }^{6}$ Epidemiology and Biostatistics, and ${ }^{7}$ Endoscopy, European Institute of Oncology, I-20141 Milan, Italy; ${ }^{8}$ Department of Biology and Biotechnology, University of Pavia, I-27100 Pavia, Italy

Received August 10, 2016; Accepted January 17, 2017

DOI: $10.3892 / 01.2017 .6354$

\begin{abstract}
Hereditary diffuse gastric cancer (HDGC) is associated with E-cadherin $1(\mathrm{CDH})$ germline mutations. In the present study, two unusual HDGC cases are described. Case 1 was a female with no family history of gastric cancer who developed Hodgkin's lymphoma at 19 years of age, and DGC at 32 years of age. Due to her young age (32 years), the patient was examined for $\mathrm{CDH} 1$ abnormalities and a deleterious mutation was identified. Her father and younger sister were identified to be carriers of the mutation. Case 2 was a 36-year-old female diagnosed with lobular breast cancer (LBC); her mother had LBC, and her grandmother had LBC and DGC. The molecular test was wild-type for breast cancer susceptibility genes 1 and 2; however, a large deletion in $\mathrm{CDH}$ was identified. At prophylactic gastrectomy, early DGC was identified. Early onset of DGC and LBC justifies testing for $C D H 1$. A better knowledge of tumor natural history in carrier subjects is important to aid genetic counseling, in order to assess the surveillance time required prior to carrying out prophylactic surgery.
\end{abstract}

\section{Introduction}

Gastric cancer (GC) (1) is divided, according to the classification of Lauren (2), into intestinal, diffuse and mixed subtypes. In Western countries, GC has exhibited a decreased incidence primarily as a result of improvements in hygiene and food conservation in the last 50 years (3). However, this decline holds true essentially for the intestinal histotype only, whereas the incidence rate of diffuse GC (DGC) is relatively stable (1).

Correspondence to: Mrs. Irene Feroce, Division of Cancer Prevention and Genetics, European Institute of Oncology, $435 \mathrm{Via}$ Ripamonti, I-20141 Milan, Italy

E-mail: irene.feroce@ieo.it

Key words: diffuse gastric cancer, lobular breast cancer, cadherin 1 , signet ring gastric cancer, genetic counseling
Intestinal GC is generally associated with chronic inflammation, and is frequently associated with chronic infection by Helicobacter pylori (4). Conversely, DGC is not associated with chronic inflammation. This is certainly the case for patients with hereditary DGC (HDGC) (5).

HDGC syndrome is defined on the basis of family history of DGC, early onset of the disease or presence of DGC associated with lobular breast cancer (LBC). The genetic alteration linked to this syndrome is a germline mutation of the E-cadherin $1(\mathrm{CDH})$ gene. Its product, endothelial cadherin, is an adhesion molecule crucial for maintaining epithelial cell polarity and architectural structure (6). The syndrome is characterized by an autosomal dominant transmission and requires a second 'hit' (mutation, loss of heterozygosity, methylation) to inactivate the wild-type allele (7-9).

Hereditary cancer syndromes are rare compared with sporadic cancer, but their investigation is crucial, due to different clinical management that mutation carriers warrant and the implications for the disease-free relatives (6).

In the present study, two families are described who were identified in a screening and genetic counseling program for HDGC.

\section{Case report}

Subjects. Subjects who matched the HDGC condition based on the criteria of Brooks-Wilson et al (10) and agreed to undergo genetic testing signed an informed consent declaration and received genetic counseling.

Molecular characterization. A mutational screening of the CDH1 gene was previously carried out by complementary molecular approaches including DNA sequencing, multiplex ligation-dependent probe amplification, single-nucleotide primer extension, bisulfite sequencing, reverse transcription-polymerase chain reaction and bioinformatics tools (11). During that screening, a c.688-1G $>C$ mutation affecting mRNA splicing and leading to a premature termination codon were identified in case 1 , whereas an intragenic deletion of exons 7 and 8 were identified in case 2 (11).

Pathology. Biopsy and surgical specimens were fixed in $10 \%$ neutral-buffered formalin (DDK Italia, Milan, Italy), 
equivalent to $\sim 4 \%$ formaldehyde solution for $1-5 \mathrm{~h}$ at room temperature and subsequently for another $5 \mathrm{~h}$ at $37^{\circ} \mathrm{C}$. Following fixation, tissue samples were dehydrated in an ethanol/xylene series and embedded using fresh paraffin wax maintained at $55-60^{\circ} \mathrm{C}$. Once embedded in paraffin wax, samples were stored at room temperature $\left(20-25^{\circ} \mathrm{C}\right)$ prior to sectioning. Consecutive serial sections $(4 \mu \mathrm{m})$ were cut from the tissue block immediately prior to testing. Sections were mounted on slides and dried for 12-24 h at room temperature or $1 \mathrm{~h}$ at $60^{\circ} \mathrm{C}$. Sections were subsequently deparaffinized and rehydrated in a xylene/ethanol series. Histology was confirmed with hematoxylin and eosin staining. Briefly, hematoxylin and eosin staining was performed using a semi-automated instrument Tissue-Tek Sakura (Sakura Finetek Europe B.V., Flemingweg, Netherlands) using hematoxylin obtained from Bio Optica Milano SpA (Milan, Italy) and eosin obtained from BDH Analytical Chemicals (Radnor, PA, USA).

Case 1. A 32 years old female was diagnosed with Hodgkin's lymphoma at 19 years of age, and was treated with mantle radiotherapy and chemotherapy (Adriamycin, bleomycin, vinblastine and dacarbazine), with no relapses. At 32 years of age, she received a diagnosis of DGC and was treated with total gastrectomy [final stage pT3 pN1 G4 (p, pathology; T, tumor; N, node; G, grade)] followed by chemotherapy with 6 cycles of capecitabine and oxaliplatin. A local relapse was detected using computed tomography, 18 months following chemotherapy. The patient was treated with a surgical resection of the left colic flexure, surrenectomy and splenectomy. A second line of chemotherapy was initiated with 8 cycles of irinotecan and capecitabine. Even though the pedigree was silent at that time, due to her young age, she was offered genetic counseling and was tested for $C D H 1$. The results revealed a pathogenic splicing mutation (11). As there was no family history of gastric cancer, her parents were tested. Her 63-year-old father was identified to be a carrier of the same mutation; subsequently, her 30-year-old sister was tested and was also found to be a carrier of the same mutation. Both relatives underwent gastroscopy, according to the gastroscopy protocol for mutation carriers, which included 36 random biopsies, 6 for each gastric anatomical region. All of the father's tissue samples were negative, whereas the sister had a positive sample for signet ring gastric cancer (SRGC). She underwent total gastrectomy with a subsequent diagnosis of SRGC of the cardia and gastric body (pTla pN0 G3). The father elected to monitor the stomach annually instead of undergoing a prophylactic gastrectomy. At his second endoscopic assessment, one biopsy sample was identified to be positive for SRGC. At the final pathological report following total gastrectomy, DGC of pT1a pN0 G2 of the fundus was identified (Fig. 1).

The sisters were included in an intensive breast-screening program with mammography ultrasound and magnetic resonance imaging.

Case 2. A 36-year-old female was diagnosed with LBC at 36 years of age, and underwent a nipple-sparing mastectomy. The final pathological staging was pT1c pN0 G2 LBC, estrogen- and progesterone-receptor positive, human epidermal growth factor $2 /$ neu-negative and $15 \%$ proliferation marker protein $\mathrm{Ki}-67$. As adjuvant treatment, the patient received 5 years of tamoxifen and ovarian suppression with luteinizing hormone-releasing hormone analogue. Due to the patient's young age and family history (her mother and grandmother were also diagnosed with LBC at 45 and 62 years of age, respectively), she was offered genetic counseling and tested for breast cancer susceptibility genes 1 and 2, which were shown to be wild-type. As her maternal grandmother was diagnosed with DGC at 64 years of age, documented with a pathological report, a $C D H 1$ molecular test was performed. This test revealed a pathogenic deletion of exons 7-8 of CDHI (11). The patient underwent gastroscopy with 36 random biopsies, all of which were negative. Following genetic counseling, she decided to undergo prophylactic gastrectomy. The final pathological report post-surgery was positive for a small SRGC pT1a pN0 G3. The family history suggested that the mutation was inherited from the mother's side (the mother was already deceased). The molecular test was proposed to the brother and the maternal uncle. Results identified that each of these relatives were carriers of the same deletion. The 41-year-old brother underwent a gastroscopy that exhibited positivity for SRGC, with a final post-total gastrectomy staging of pT1a pN0 G3 (Fig. 2). The patient's 69-year-old uncle was completely asymptomatic and declined to undergo gastroscopy. At a subsequent follow-up visit by the female patient, the proband was diagnosed with a contralateral LBC and the patient underwent a mastectomy with a pathological report of a pT1c pN0 G2 LBC.

\section{Discussion}

Families in whom a deleterious genetic mutation runs require genetic counseling, clinical surveillance and the possible recommendation of prophylactic surgery. HDGC is characterized by a lifetime DGC risk of $80 \%$ in males and $90 \%$ in females, combining the risk of DGC and LBC $(12,13)$.

In Case 1, the family is notably similar to another case where a different de novo $\mathrm{CDH} 1$ mutation was reported and the proband also exhibited a previous Hodgkin's lymphoma (14). Although conclusions cannot be drawn from only two cases, it may be hypothesized that HDGC has an increased sensitivity to radiation damage, with rapid onset of the disease after radiotherapy, a condition well documented in Li-Fraumeni syndrome (15).

In Case 2, an essential point is the degree of thoroughness warranted when reconstructing family history in order to perform the appropriate molecular tests. Indeed, it is increasingly evident that LBC can be developed in carriers of the $C D H 1$ mutation $(16,17)$.

A number of issues require addressing. One is the role of screening gastroscopy, as the nature of DGC makes it difficult to observe cancer lesions at the gastric mucosa level, and multiple random biopsies may not detect an early GC. On the other hand, it is not clear when these early lesions may progress to the point where they become clinically relevant. This issue is crucial and should be comprehensively examined, in order to better counsel whether and when to pursue prophylactic surgery. Furthermore, the time between the 
$\mathbf{A}$

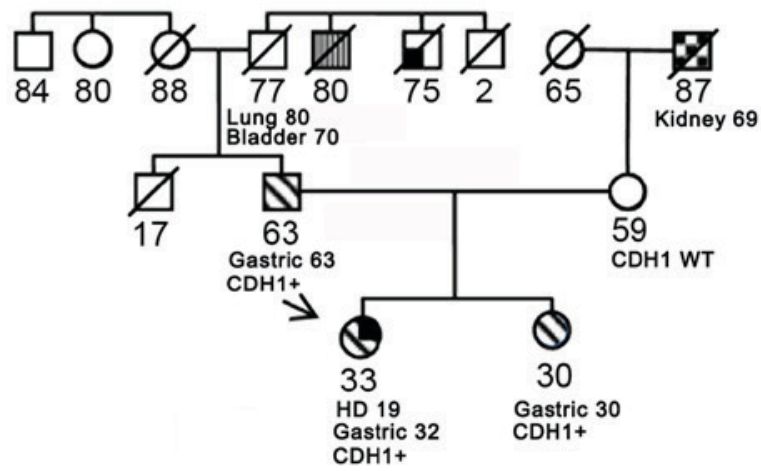

B

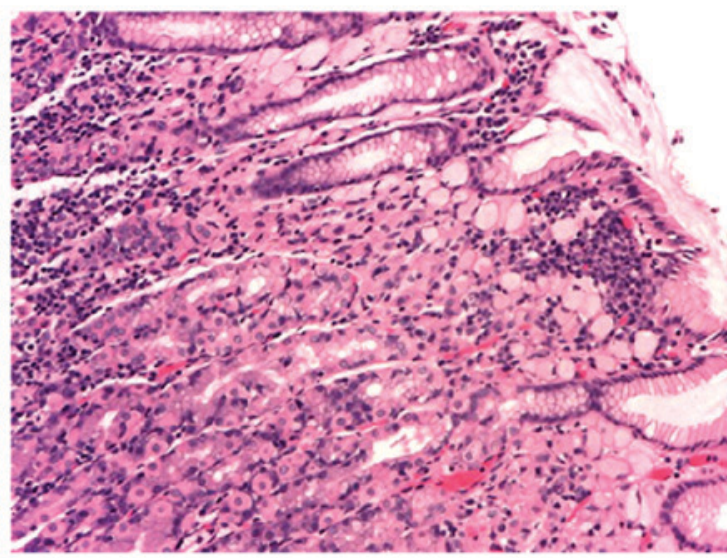

Figure 1. (A) Case 1 family tree and the cancer histology of the proband. The arrow indicates the patient. Numbers indicate age (in years) at present, at diagnosis with the cancer type stated or at mortality. (B) Hematoxylin and eosin staining of gastric tissue. Magnification, x200. Signet ring cell carcinoma arising in this stomach is limited to the lamina propria. The neoplastic cells are easily recognizable because of their typical features consisting of a mucin-replete cytoplasm pushing the nucleus to the periphery of the cell. CDH1+, mutation currier subject; WT, wild-type; HD, Hodgkin's disease.

$\mathbf{A}$

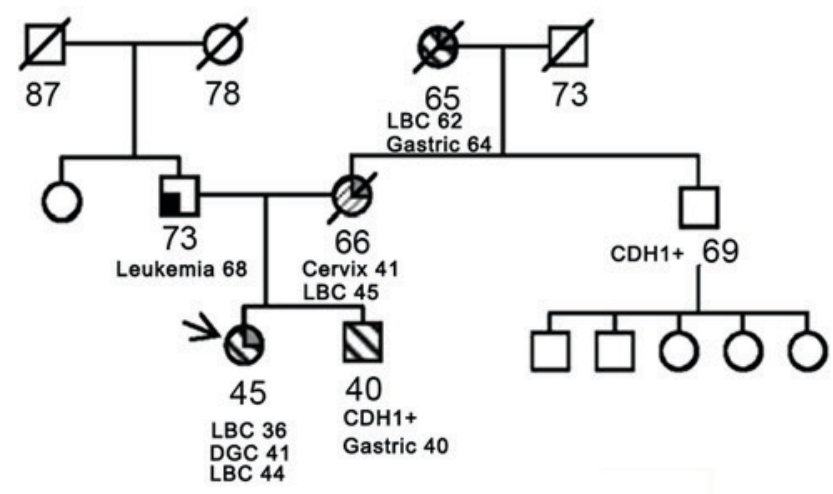

Family 2

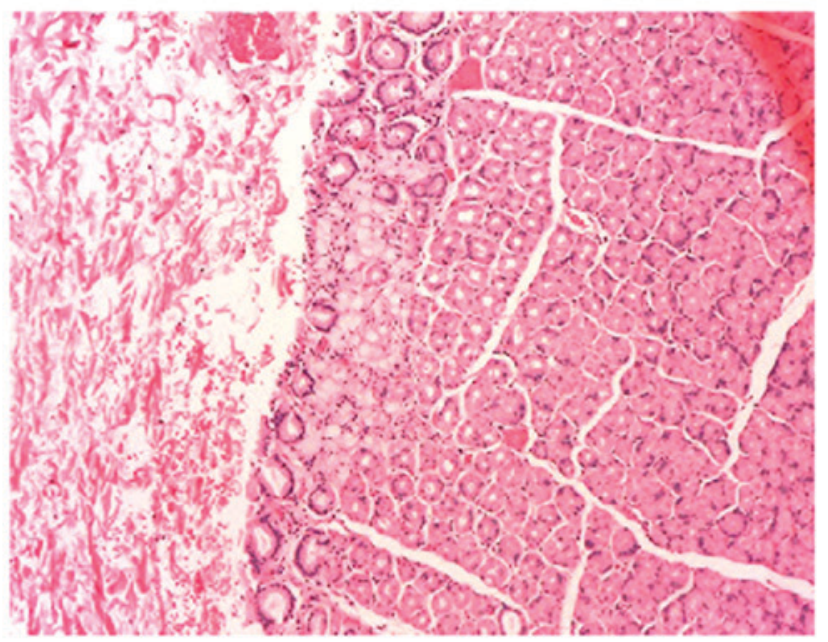

Figure 2. (A) Case 2 family tree and the cancer histology of the proband. The arrow indicates the patient. Numbers indicate age (in years) at present, at diagnosis with the cancer type stated or at mortality. (B) Hematoxylin and eosin staining of gastric tissue. Magnification, x200. Signet ring cell carcinoma arising in this stomach is limited to the mucosa. $\mathrm{CDH1}+$, mutation currier subject; $\mathrm{LBC}$, lobular breast cancer; DGC, diffuse gastric cancer.

initiation of the carcinogenesis process and clinically relevant cancer may allow chemopreventive strategies to be developed and undertaken, even though, compared with other organs, far fewer studies have been performed and few hypotheses have been drawn which focus on sporadic GC $(18,19)$. Another issue is the prognosis of these tumors. It has been suggested that a generic family history for GC may define a less aggressive cancer, compared with the pure sporadic cancer $(20,21)$; however, recently, overall survival rate of $C D H 1$ mutation carriers has been indicated to be worse compared with patients exhibiting wild-type $C D H 1$, with 4 vs. $13 \%$ for 5 -year survival rate (22).

On the basis of the two cases reported in the present study, the importance of young age at cancer diagnosis should be underlined, even in a family with no known history of cancer, and genetic testing for LBC must be considered, even though the proband may not meet the most stringent selection criteria $(10,17,22)$.

A better knowledge of the natural history of diseases that develop in $\mathrm{CDH} 1$ carriers may assist in improving the counseling process and the development of preventive strategies in order to delay, or even circumvent, prophylactic gastrectomy or any other prophylactic surgery.

\section{Acknowledgements}

The authors of the present study would like to acknowledge support from the Italian Ministry of Health. M.M. was a recipient of a Giovanni Magni postdoctoral fellowship from the Adriano Buzzati-Traverso Foundation. 


\section{References}

1. Crew KD and Neugut AI: Epidemiology of gastric cancer. World J Gastroenterol 12: 354-362, 2006.

2. Lauren P: The two histological main types of gastric carcinoma: Diffuse and so-called intestinal-type carcinoma. An attempt at a histo-clinical classification. Acta Pathol Microbiol Scand 64: 31-49, 1965.

3. Howson CP, Hiyama T and Wynder EL: The decline in gastric cancer: Epidemiology of an unplanned triumph. Epidemiol Rev 8: 1-27, 1986.

4. Sepulveda AR: Helicobacter, Inflammation, and Gastric Cancer. Curr Pathobiol Rep 1: 9-18, 2013.

5. Yakirevich E and Resnick MB: Pathology of gastric cancer and its precursor lesions. Gastroenterol Clin North Am 42: 261-284, 2013.

6. Kluijt I, Siemerink EJ, Ausems MG, van Os TA, de Jong D, Simões-Correia J, van Krieken JH, Ligtenberg MJ, Figueiredo J, van Riel E, et al: CDH1-related hereditary diffuse gastric cancer syndrome: Clinical variations and implications for counseling. Int J Cancer 131: 367-376, 2012.

7. Humar B, Blair V, Charlton A, More H, Martin I and Guilford P: E-cadherin deficiency initiates gastric signet-ring cell carcinoma in mice and man. Cancer Res 69: 2050-2056, 2009.

8. Oliveira C, Sousa S, Pinheiro H, Karam R, Bordeira-Carrico R, Senz J, Kaurah P, Carvalho J, Pereira R, Gusmão L, et al: Quantification of epigenetic and genetic 2nd hits in CDH1 during hereditary diffuse gastric cancer syndrome progression. Gastroenterology 136: 2137-2148, 2009.

9. Nakayama S, Sasaki A, Mese H, Alcalde RE, Tsuji T and Matsumura T: The E-cadherin gene is silenced by $\mathrm{CpG}$ methylation in human oral squamous cell carcinomas. Int J Cancer 93: 667-673, 2001.

10. Brooks-Wilson AR, Kaurah P, Suriano G, Leach S, Senz J, Grehan N, Butterfield YS, Jeyes J, Schinas J, Bacani J, et al: Germline E-cadherin mutations in hereditary diffuse gastric cancer: Assessment of 42 new families and review of genetic screening criteria. J Med Genet 41: 508-517, 2004.

11. Molinaro V, Pensotti V, Marabelli M, Feroce I, Barile M, Pozzi S, Laghi L, Serrano D, Bernard L, Bonanni B and Ranzani GN: Complementary molecular approaches reveal heterogeneous CDH1 germline defects in Italian patients with hereditary diffuse gastric cancer (HDGC) syndrome. Genes Chromosomes Cancer 53: 432-445, 2014.

12. Pharoah PD, Guilford P and Caldas C; International Gastric Cancer Linkage Consortium: Incidence of gastric cancer and breast cancer in CDH1 (E-cadherin) mutation carriers from hereditary diffuse gastric cancer families. Gastroenterology 121 : $1348-1353,2001$.
13. Kaurah P, MacMillan A, Boyd N, Senz J, De Luca A, Chun N, Suriano G, Zaor S, Van Manen L, Gilpin C, et al: Founder and recurrent $\mathrm{CDH} 1$ mutations in families with hereditary diffuse gastric cancer. JAMA 297: 2360-2372, 2007.

14. Shah MA, Salo-Mullen E, Stadler Z, Ruggeri JM, Mirander M, Pristyazhnyuk Y and Zhang L: De novo CDH1 mutation in a family presenting with early-onset diffuse gastric cancer. Clin Genet 82: 283-287, 2012.

15. Salmon A, Amikam D, Sodha N, Davidson S, Basel-Vanagaite L, Eeles RA, Abeliovich D and Peretz T: Rapid development of post-radiotherapy sarcoma and breast cancer in a patient with a novel germline 'de-novo' TP53 mutation. Clin Oncol (R Coll Radiol) 19: 490-493, 2007.

16. McVeigh TP, Choi JK, Miller NM, Green AJ and Kerin MJ: Lobular breast cancer in a CDH1 splice site mutation carrier: Case report and review of the literature. Clin Breast Cancer 14: e47-e51, 2014.

17. Corso G, Figueiredo J, Biffi R, Trentin C, Bonanni B, Feroce I, Serrano D, Cassano E, Annibale B, Melo S, et al: E-cadherin germline mutation carriers: Clinical management and genetic implications. Cancer Metastasis Rev 33: 1081-1094, 2014.

18. Tan VP and Wong BC: Gastric cancer chemoprevention: The current evidence. Gastroenterol Clin North Am 42: 299-316, 2013.

19. Yadav SK, Sah AK, Jha RK, Sah P and Shah DK: Turmeric (curcumin) remedies gastroprotective action. Pharmacogn Rev 7: 42-46, 2013.

20. Yatsuya H, Toyoshima H, Mizoue T, Kondo T, Tamakoshi K, Hori Y, Tokui N, Hoshiyama Y, Kikuchi S, Sakata K, et al: Family history and the risk of stomach cancer death in Japan: Differences by age and gender. Int J Cancer 97: 688-694, 2002.

21. Han MA, Oh MG, Choi IJ, Park SR, Ryu KW, Nam BH, Cho SJ, Kim CG, Lee JH and Kim YW: Association of family history with cancer recurrence and survival in patients with gastric cancer. J Clin Oncol 30: 701-708, 2012.

22. van der Post RS, Vogelaar IP, Manders P, van der Kolk LE, Cats A, van Hest LP, Sijmons R, Aalfs CM, Ausems MG, Gómez Garcia EB, et al: Accuracy of hereditary diffuse gastric cancer testing criteria and outcomes in patients with a germline mutation in CDH1. Gastroenterology 149: 897-906.e19, 2015. 